\title{
Loi de Benford, relations de récurrence et suites équidistribuées
}

\author{
Paul Jolissaint \\ Paul Jolissaint studied physics and mathematics at the University of Geneva where \\ he obtained his doctoral degree in 1987. He is a part time lecturer at the Institute of \\ Mathematics of the University of Neuchâtel and a part time professor of mathematics \\ and physics at the Lycée cantonal de Porrentruy (Jura, CH). His main interests are von \\ Neumann algebras, harmonic analysis and actions of groups.
}

\section{Introduction}

Lorsque l'on considère un ensemble de valeurs numériques relevées au hasard (cours de la Bourse, nombres extraits de journaux,...), on constate que les chiffres de 1 à 9 n'apparaissent pas avec la même fréquence comme chiffre significatif (le premier chiffre à gauche) dans l'écriture décimale de l'ensemble de valeurs étudiées: en fait, étant donné $d \in\{1, \ldots, 9\}$, la proportion de valeurs de la liste dont le chiffre significatif est $d$ est voisine de $\log (1+1 / d)$. Ainsi, la proportion de valeurs commençant par 1 est environ égale à $\log (2) \approx 0.301$, celle des valeurs commençant par 2 vaut à peu près $\log (3 / 2) \approx$ 0.176 , et ainsi de suite jusqu'à la proportion des valeurs commençant par 9 qui est de l'ordre de $\log (10 / 9) \approx 0.046$. Cette constatation fut la première fois publiée en 1881 par

Betrachtet man numerische Daten, die von verschiedensten Quellen stammen wie z.B. Aktienkurse oder Zahlenmaterial aus Zeitungsartikeln, usw., so stellt man fest, dass die Ziffern 1, . , 9 nicht mit gleicher Wahrscheinlichkeit als erste Ziffer dieser Daten auftreten. In den 30er Jahren des letzten Jahrhunderts hat der Physiker F. Benford mehr als 20000 solcher Daten gesammelt und ist dabei zur Beobachtung gelangt, dass die Ziffer $d$ mit Wahrscheinlichkeit $\approx \log _{10}(1+1 / d)$ als erste auftritt. Man kann sich nun fragen, ob dieses Gesetz auch für Zahlenfolgen $\left(a_{n}\right)_{n \geq 0}$ im Intervall [1, $\infty$ [ Gültigkeit besitzt. In der Tat lässt sich diese Frage für die Folge $a_{n}=2^{n}$ unter Anwendung von $\mathrm{H}$. Weyls Ergodensatz positiv beantworten. In dem vorliegenden Beitrag zeigt der Autor, dass eine grosse Klasse von Folgen, die durch lineare Rekursionen definiert sind, das Benfordsche Gesetz erfüllen. Als wesentliches Werkzeug wird dazu H. Weyls berühmter Satz über die „Gleichverteilung von Zahlen mod 1“ herangezogen. 
S. Newcomb dans la revue American Journal of Mathematics, mais passa complètement inaperçue. Cinquante ans plus tard, le physicien Franck Benford fait la même constatation et publie en 1938 un article dans les Proceedings of the American Philosophical Society contenant 20229 observations provenant de divers domaines. Il constate que la table correspondante des premiers chiffres s'accorde avec la loi qui portera désormais son nom. Pour davantage de détails sur la loi de Benford et son histoire, nous conseillons au lecteur de consulter l'excellent article de T. Hill [3].

Il est plus ou moins connu que cette loi est aussi vérifiée pour certaines suites de nombres telles que la suite de Fibonacci $\left(F_{n}\right)_{n \geq 0}$ ou la suite $\left(2^{n}\right)_{n \geq 0}$, par exemple. Or, de telles suites satisfont les relations de récurrence $F_{n+2}=F_{n+1}+F_{n}$ pour la suite de Fibonacci, et $a_{n+1}=2 a_{n}$ pour la seconde. A propos de cette dernière, voir [1], pages 135 et 136 ; c'est d'ailleurs cet exemple qui a motivé notre étude.

Le but de notre article est de présenter une condition suffisante pour qu'une suite de nombres $a_{n} \geq 1$ (non nécessairement entiers) satisfaisant une relation de récurrence de la forme

$$
a_{n+q}=c_{1} a_{n+q-1}+c_{2} a_{n+q-2}+\ldots+c_{q} a_{n}
$$

où $c_{1}, \ldots, c_{q} \in \mathbb{R}$ et $c_{q} \neq 0$, obéisse à la loi de Benford non seulement en base 10 mais dans d'autres bases $b \geq 3$. (Notons que toute suite $\left(a_{n}\right) \subset[1, \infty$ [ satisfait trivialement la loi de Benford en base 2.)

Avant d'énoncer le résultat précis, nous avons besoin de quelques rappels et de fixer nos notations.

Si $E$ désigne un ensemble fini, on note $|E|$ son cardinal. Si $x$ est un nombre réel, on note $[x]$ sa partie entière et $\{x\}$ sa partie fractionnaire: $[x]$ est le plus grand entier inférieur ou égal à $x$, et $\{x\}=x-[x]$. Soit maintenant $b$ un entier $\geq 3$. Puisque l'intervalle $[1,+\infty[$ est la réunion disjointe

$$
\left[1,+\infty\left[=\bigsqcup_{r \geq 0}^{b-1} \bigsqcup_{d=1}^{b}\left[d \cdot b^{r},(d+1) \cdot b^{r}[,\right.\right.\right.
$$

tout nombre $a \in\left[1,+\infty\right.$ [ appartient à un unique intervalle $\left[d \cdot b^{r},(d+1) \cdot b^{r}[\right.$ où $r \geq 0$ et $1 \leq d \leq b-1$ sont entiers. Le digit $d$ est le (premier) chiffre significatif de a en base $b$. Si $\left(a_{n}\right)_{n \geq 0} \subset\left[1,+\infty\right.$ [ et $b$ sont fixés, on note $d_{n}$ le chiffre significatif de $a_{n}$ en base $b$.

Définition 1.1 La suite $\left(a_{n}\right)_{n \geq 0} \subset[1,+\infty$ [ satisfait la loi de Benford par rapport à la base $b$ si, pour tout $d \in\{1, \ldots, b-1\}$, on a

$$
\lim _{N \rightarrow \infty} \frac{1}{N}\left|\left\{n \mid 0 \leq n \leq N-1, d_{n}=d\right\}\right|=\log _{b}(1+1 / d) .
$$

Nous allons également utiliser quelques faits sur les relations de récurrence d'ordre $q$ telles que la relation (1). Récrivons-la ainsi:

$$
a_{n+q}-c_{1} a_{n+q-1}-\ldots-c_{q} a_{n}=0 .
$$


On associe à une telle relation un polynôme $p(x)$ appelé polynôme caractéristique: c'est le polynôme

$$
p(x)=x^{q}-c_{1} x^{q-1}-\ldots-c_{q} .
$$

La raison en est que toute solution $\left(a_{n}\right)_{n \geq 0}$ de la relation de récurrence s'exprime à l'aide des racines de $p(x)$ de la façon suivante: écrivons $p(x)=\left(x-\xi_{1}\right)^{\mu_{1}}\left(x-\xi_{2}\right)^{\mu_{2}} \cdots(x-$ $\left.\xi_{m}\right)^{\mu_{m}}$, où $\xi_{1}, \ldots, \xi_{m} \in \mathbb{C}$ sont les racines distinctes de $p(x)$, et, pour $1 \leq j \leq m$, l'entier $\mu_{j} \geq 1$ est l'ordre de $\xi_{j}$. Alors toute solution $\left(a_{n}\right)_{n \geq 0}$ de la relation (2) est une combinaison linéaire des suites $\left(n^{k} \xi_{j}^{n}\right)_{n \geq 0}$ pour $0 \leq k<\mu_{j}$ et $1 \leq j \leq m$. Plus précisément, il existe des constantes $\alpha_{j, k}$ déterminées par les conditions initiales $a_{0}, a_{1}, \ldots, a_{q-1}$ telles que

$$
a_{n}=\sum_{j=1}^{m} \sum_{k=0}^{\mu_{j}-1} \alpha_{j, k} n^{k} \xi_{j}^{n}
$$

pour tout $n \geq 0$.

Nous sommes prêts à énoncer le résultat principal de l'article:

Théorème 1.2 Soit $p(x)=x^{q}-c_{1} x^{q-1}-\ldots-c_{q-1} x-c_{q}$ un polynôme de degré $q$ qui possède une racine $\xi>1$ de multiplicité 1 telle que $|\eta|<\xi$ pour toute autre racine $\eta$ de $p(x)$. Soit $\left(a_{n}\right)_{n \geq 0} \subset[1,+\infty[$ une suite satisfaisant la relation de récurrence associée $a_{n+q}-c_{1} a_{n+q-1}-\ldots-c_{q} a_{n}=0$ et telle que

$$
\inf \left\{\frac{a_{n}}{\xi^{n}} \mid n \geq 0\right\}>0 .
$$

Si $b \geq 3$ est un entier tel que $\log _{b}(\xi)$ est irrationel, alors la suite $\left(a_{n}\right)$ satisfait la loi de Benford par rapport à $b$, et il en est de même de toute sous-suite $\left(a_{Q(n)}\right)_{n \geq 0}$ où $Q(x)$ est un polynôme non constant à coefficients entiers tel que $Q(n) \geq 0$ pour tout entier $n \geq 0$.

Remarque. Observons que la condition (5) est indispensable: en effet, la suite $\left(a_{n}\right)$ définie par $a_{n}=4^{n}$ satisfait la relation de récurrence

$$
a_{n+2}=9 a_{n+1}-20 a_{n},
$$

les racines du polynôme caractéristique associé sont 4 et 5, et elle ne satisfait évidemment pas la loi de Benford pour $b=4$ bien que $\log _{4}(5)$ soit irrationnel.

La preuve du théorème s'articule ainsi: le polynôme $Q(x)$ étant fixé, on associe à la suite $\left(a_{Q(n)}\right)_{n \geq 0}$ la suite $\left(\lambda_{n}\right)_{n \geq 0} \subset\left[0,1\left[\right.\right.$ définie par: $\lambda_{n}=\left\{\log _{b}\left(a_{Q(n)}\right)\right\}=\log _{b}\left(a_{Q(n)}\right)-$ $\left[\log _{b}\left(a_{Q(n)}\right)\right]$. On montre d'abord que si cette dernière est équidistribuée dans $[0,1]$ (voir le paragraphe 3 ), alors $\left(a_{Q(n)}\right)$ satisfait la loi de Benford par rapport à $b$; ensuite, grâce à un théorème de Weyl [5] (théorème 2.4 ci-après), les hypothèses du théorème 1.2 assurent que $\left(\lambda_{n}\right)_{n \geq 0}$ est équidistribuée dans $[0,1]$. La preuve du théorème 1.2 est donnée dans le paragraphe 3 , et le paragraphe 4 est consacré à quelques exemples. 


\section{Les suites équidistribuées}

Nous commençons par rappeler la notion de suite équidistribuée:

Définition 2.1 Une suite $\left(u_{n}\right)_{n \geq 0}$ contenue dans l'intervalle [0,1] est équidistribuée si, pour tous $0 \leq a<b \leq 1$, on a

$$
\lim _{N \rightarrow \infty} \frac{1}{N}\left|\left\{n \mid 0 \leq n \leq N-1, a \leq u_{n}<b\right\}\right|=b-a .
$$

Autrement dit, une suite équidistribuée se répartit très uniformément dans chaque sousintervalle de $[0,1]$. Cette notion est due à $\mathrm{H}$. Weyl [5], ainsi que le critère suivant qui permet de donner des exemples de telles suites.

Théorème 2.2 (H. Weyl) Pour une suite $\left(u_{n}\right)_{n \geq 0}$ contenue dans l'intervalle $[0,1]$, les conditions suivantes sont équivalentes:

(1) $\left(u_{n}\right)$ est équidistribuée ;

(2) pour toute fonction continue (à valeurs réelles ou complexes) $f$ sur $[0,1]$ telle que $f(0)=f(1)$, on a

$$
\int_{0}^{1} f(x) d x=\lim _{N \rightarrow \infty} \frac{1}{N} \sum_{n=0}^{N-1} f\left(u_{n}\right)
$$

(3) pour tout entier $\ell \neq 0$,

$$
\lim _{N \rightarrow \infty} \frac{1}{N} \sum_{n=0}^{N-1} e^{2 \pi i \ell u_{n}}=0 .
$$

La preuve est exposée notamment dans [1] et dans [4]. En guise d'application, rappelons l'exemple classique:

Exemple 2.3 Soit $\xi \in \mathbb{R}$ un nombre irrationnel et soit $u_{n}=n \xi-[n \xi]$ la suite des parties fractionnaires de la suite $(n \xi)_{n \geq 0}$. Alors $\left(u_{n}\right)_{n \geq 0}$ est équidistribuée.

Preuve. La condition (3) du théorème est particulièrement bien adaptée à ce cas. Fixons donc un entier $\ell \neq 0$ et posons $z=e^{2 \pi i \ell \xi}$. Observons que $z \neq 1$ quel que soit $\ell \neq 0$ puisque $\xi$ est irrationnel.

Comme $e^{2 \pi i \ell u_{n}}=e^{2 \pi i \ell n \xi}=z^{n}$ pour tout $n$, on a:

$$
\frac{1}{N} \sum_{n=0}^{N-1} e^{2 \pi i \ell u_{n}}=\frac{1}{N} \sum_{n=0}^{N-1} z^{n}=\frac{1}{N} \frac{z^{N}-1}{z-1} \rightarrow 0
$$

lorsque $N \rightarrow \infty$.

La preuve du théorème 1.2 utilise la généralisation suivante de l'exemple ci-dessus dont la preuve est nettement plus complexe (voir [5] et le théorème 21 de [2]): 
Théorème 2.4 (H. Weyl) Soit $R(x)=r_{m} x^{m}+r_{m-1} x^{m-1}+\ldots+r_{1} x+r_{0}$ un polynôme à coefficients réels avec $r_{m} \neq 0$ et $m>0$ tel que l'un au moins des $r_{i}$ soit irrationnel parmi $r_{1}, \ldots, r_{m}$. Alors la suite des parties fractionnaires $(\{R(n)\})_{n \geq 0}$ est équidistribuée.

Voyons maintenant quelle est la relation entre une suite $\left(a_{n}\right) \subset[1, \infty$ [ qui satisfait la loi de Benford par rapport à une base $b$ et les suites équidistribuées dans $[0,1]$.

Proposition 2.5 Soit $\left(a_{n}\right)_{n \geq 0} \subset[1,+\infty[$ et soit $b \geq 3$ un entier. Posons

$$
\lambda_{n}=\log _{b}\left(a_{n}\right)-\left[\log _{b}\left(a_{n}\right)\right] \in[0,1]
$$

pour tout $n \geq 0$. Si la suite $\left(\lambda_{n}\right)_{n \geq 0}$ est équidistribuée alors la suite $\left(a_{n}\right)_{n \geq 0}$ satisfait la loi de Benford par rapport à $b$.

Preuve. Comme dans le paragraphe précédent, notons $d_{n}$ le chiffre significatif de $a_{n}$ en base $b$. Il existe un entier $r=r_{n} \geq 0$ tel que

$$
d_{n} \cdot b^{r} \leq a_{n}<\left(d_{n}+1\right) \cdot b^{r}
$$

ceci est équivalent à

$$
\log _{b}\left(d_{n}\right) \leq \log _{b}\left(a_{n}\right)-r<\log _{b}\left(d_{n}+1\right)
$$

Cela montre entre autres que $r$ est égal à $\left[\log _{b}\left(a_{n}\right)\right]$. Ainsi, si $d \in\{1, \ldots, b-1\}$ est fixé, on a $d_{n}=d$ si et seulement si $\lambda_{n} \in\left[\log _{b}(d), \log _{b}(d+1)\left[\right.\right.$. Si $\left(\lambda_{n}\right)$ est équidistribuée, on obtient:

$$
\begin{aligned}
\frac{1}{N}\left|\left\{n \mid 0 \leq n \leq N-1, d_{n}=d\right\}\right| & \\
& =\frac{1}{N} \mid\left\{n \mid 0 \leq n \leq N-1, \lambda_{n} \in\left[\log _{b}(d), \log _{b}(d+1)[\} \mid\right.\right. \\
& \rightarrow \log _{b}(d+1)-\log _{b}(d)=\log _{b}(1+1 / d)
\end{aligned}
$$

lorsque $N \rightarrow \infty$.

Cela achève la preuve de la proposition.

\section{Preuve du théorème 1.2}

Rappelons les hypothèses: $\left(a_{n}\right)_{n \geq 0} \subset[1,+\infty$ [ est une suite qui satisfait la relation de récurrence

$$
a_{n+q}-c_{1} a_{n+q-1}-\ldots-c_{q} a_{n}=0 .
$$

Soient $\xi=\xi_{1}>1$ et $\xi_{2}, \ldots, \xi_{m}$ les racines de son polynôme caractéristique. On suppose que $\left|\xi_{j}\right|<\xi$ pour $j \geq 2$ et que $\inf _{n} \frac{a_{n}}{\xi^{n}}>0$. 
Ecrivons, compte tenu des rappels du paragraphe 1 (formule (4)):

$$
a_{n}=\alpha \xi^{n}+\sum_{j=2}^{m} \sum_{k=0}^{\mu_{j}-1} \alpha_{j, k} n^{k} \xi_{j}^{n}
$$

pour $n \geq 0$. Récrivons (7) ainsi:

$$
a_{n}=\xi^{n}\left(\alpha+\sum_{j=2}^{m} \sum_{k=0}^{\mu_{j}-1} \alpha_{j, k} n^{k}\left(\frac{\xi_{j}}{\xi}\right)^{n}\right)
$$

D'après les hypothèses, $\alpha>0$ et l'expression entre parenthèses aussi. Soit $Q(x)=b_{s} x^{s}+$ $b_{s-1} x^{s-1}+\ldots+b_{1} x+b_{0}$ avec $s \geq 1, b_{j} \in \mathbb{Z}, b_{s}>0$, tels que $Q(n) \geq 0$ pour tout entier $n \geq 0$. On obtient alors

$$
a_{Q(n)}=\xi^{Q(n)}\left(\alpha+\sum_{j=2}^{m} \sum_{k=0}^{\mu_{j}-1} \alpha_{j, k} Q(n)^{k}\left(\frac{\xi_{j}}{\xi}\right)^{Q(n)}\right) .
$$

D'après la proposition 2.5, il suffit de vérifier que la suite $\left(\left\{\log _{b}\left(a_{Q(n)}\right)\right\}\right)_{n \geq 0}$ est équidistribuée. Or,

$$
\log _{b}\left(a_{Q(n)}\right)=Q(n) \log _{b}(\xi)+\eta_{n}
$$

où $\eta_{n}=\log _{b}\left(\alpha+\sum_{j=2}^{m} \sum_{k=0}^{\mu_{j}-1} \alpha_{j, k} Q(n)^{k}\left(\frac{\xi_{j}}{\xi}\right)^{Q(n)}\right) \rightarrow \eta=\log _{b}(\alpha)$ lorsque $n \rightarrow \infty$.

Afin d'obtenir la conclusion, nous allons utiliser le lemme suivant:

Lemme 3.1 Soient $\left(u_{n}\right)_{n \geq 0}$ et $\left(v_{n}\right)_{n \geq 0}$ deux suites de nombres complexes telles que

(a) $\left(u_{n}\right)$ converge vers $u \in \mathbb{C}$;

(b) $\left(v_{n}\right)$ est bornée et $\lim _{N \rightarrow \infty} \frac{1}{N} \sum_{n=0}^{N-1} v_{n}=v \in \mathbb{C}$.

Alors

$$
\lim _{N \rightarrow \infty} \frac{1}{N} \sum_{n=0}^{N-1} u_{n} v_{n}=u v
$$

Preuve. Puisque $\left|u_{n}-u\right|$ converge vers 0 lorsque $n \rightarrow \infty$, elle converge également au sens de Césaro:

$$
\lim _{N \rightarrow \infty} \frac{1}{N} \sum_{n=0}^{N-1}\left|u_{n}-u\right|=0 .
$$


Soit encore $C>0$ tel que $\left|v_{n}\right| \leq C$ pour tout $n$. En utilisant les majorations suivantes:

$$
\begin{aligned}
\left|\frac{1}{N} \sum_{n=0}^{N-1} u_{n} v_{n}-u v\right| & =\left|\frac{1}{N} \sum_{n=0}^{N-1}\left(u_{n}-u\right) v_{n}+\frac{u}{N} \sum_{n=0}^{N-1} v_{n}-u v\right| \\
& \leq \frac{C}{N} \sum_{n=0}^{N-1}\left|u_{n}-u\right|+|u|\left|\frac{1}{N} \sum_{n=0}^{N-1} v_{n}-v\right|
\end{aligned}
$$

on obtient le résultat.

Pour terminer la preuve du théorème, fixons un entier $\ell \neq 0$ et posons $u_{n}=e^{2 \pi i \ell \eta_{n}}$ et $v_{n}=e^{2 \pi i \ell Q(n) \log _{b}(\xi)}$. Puisque $\log _{b}(\xi)$ est irrationnel et que $Q(x)$ est à coefficients entiers, on a, par le théorème 2.4:

$$
\lim _{N \rightarrow \infty} \frac{1}{N} \sum_{n=0}^{N-1} v_{n}=0
$$

donc

$$
\lim _{N \rightarrow \infty} \frac{1}{N} \sum_{n=0}^{N-1} u_{n} v_{n}=0,
$$

et le théorème 2.2 implique que la suite $\left(\log _{b}\left(a_{Q(n)}\right)_{n \geq 0}\right.$ est équidistribuée. Ceci achève la preuve du théorème 1.2 .

Remarques. (1) En fait, l'exemple 2.3 suffit à démontrer que la suite $\left(a_{n}\right)$ elle-même satisfait la loi de Benford.

(2) $\mathrm{Si}\left(a_{n}\right)_{n \geq 0}$ satisfait les hypothèses du théorème 1.2, certaines suites associées satisfont également la loi de Benford. C'est le cas par exemple des suites de la forme

$$
s_{n}=a_{0}+a_{1}+\ldots+a_{n} .
$$

En effet, il est facile de vérifier que si $a_{n}$ est donnée par (4) alors

$$
s_{n}=\alpha \frac{\xi^{n+1}-1}{\xi-1}+\sum_{j=2}^{m} \sum_{k=0}^{\mu_{j}-1} \alpha_{j, k} \sum_{l=0}^{n} l^{k} \xi_{j}^{l}
$$

satisfait la loi de Benford pour les mêmes valeurs de $b$, de même que toutes les sous-suites $\left(s_{Q(n)}\right)_{n \geq 0}$ comme dans le théorème 1.2.

\section{Exemples}

Nous allons présenter quatre exemples pour illustrer le théorème 1.2. Les trois derniers s'appuient sur le résultat suivant, qui nécessite quelques rappels. Un nombre $\xi$ est algébrique $(\operatorname{sur} \mathbb{Q})$ s'il est racine d'un polynôme (non identiquement nul) à coefficients dans $\mathbb{Q}$. Si c'est le cas, il existe un unique polynôme $m(x)=x^{n}+\alpha_{n-1} x^{n-1}+\ldots+\alpha_{1} x+\alpha_{0}$ à coefficients rationnels tel que 
(1) $m(\xi)=0$;

(2) si $s(x)$ est un polynôme à coefficients dans $\mathbb{Q}$ tel que $s(\xi)=0$, alors $m(x)$ divise $s(x)$.

On appelle $m(x)$ le polynôme minimal de $\xi$, et le degré de $m(x)$ s'appelle le degré de $\xi$. Rappelons également que $\xi$ est nécessairement une racine simple de $m(x)$ : en effet, si ce n'était pas le cas, $\xi$ serait aussi une racine du polynôme dérivé $m^{\prime}(x)$, qui est encore à coefficients rationnels et de degré inférieur à celui de $m(x)$.

Proposition 4.1 Soit $\xi>1$ un nombre irrationnel algébrique dont $p(x)=x^{q}-c_{1} x^{q-1}-$ $\ldots-c_{q}$ est le polynôme minimal, avec $c_{i} \in \mathbb{Q}$ pour tout $i$. Si toute autre racine $\eta$ de $p(x)$ satisfait $|\eta|<\xi$, alors $\xi^{n}$ est irrationnel pour tout entier positif n. En particulier, $\log _{b}(\xi)$ est irrationnel pour tout entier $b \geq 3$.

Preuve. Supposons par l'absurde qu'il existe un entier $n \geq 2$ et un nombre $\alpha \in \mathbb{Q}$ tels que $\xi^{n}=\alpha$. Posons $s(x)=x^{n}-\alpha$. Ses racines sont les nombres complexes $\sqrt[n]{\alpha} e^{2 i \pi k / n}$, $0 \leq k \leq n-1$, dont le module est $\sqrt[n]{\alpha}$ pour tout $k$. Comme $p(x)$ divise $s(x)$, ses racines ont toutes le même module, ce qui contredit l'hypothèse.

Exemple 4.2 (1) Soit $a_{n}=m^{n}$ pour tout $n$, où $m \geq 2$ est entier. C'est une suite qui satisfait la relation de récurrence $a_{n+1}=m a_{n}$ et $\log _{b}(m)$ est irrationnel pour tout $b$ qui n'est pas une puissance rationnelle de $m$. Comme nous l'avons indiqué dans l'introduction, cet exemple est traité dans [1] comme application du théorème de Weyl pour les valeurs $m=2$ et $b=10$.

(2) Soit $\left(F_{n}\right)_{n \geq 0}$ la suite de Fibonacci avec $F_{0}=F_{1}=1$ et $F_{n+2}=F_{n+1}+F_{n}$ pour $n \geq 0$. Son polynôme caractéristique est $p(x)=x^{2}-x-1=(x-\varphi)(x+1 / \varphi)$ où $\varphi=\frac{1}{2}(1+\sqrt{5})$ est le nombre d'or. Cette suite satisfait les conditions du théorème 1.2 et de la proposition 3.1. Donc $\left(F_{n}\right)_{n \geq 0}$ et toutes ses sous-suites $\left(F_{Q(n)}\right)$ satisfont la loi de Benford par rapport à toute base $b \geq 3$. Par ailleurs, il en est de même de la suite $\left(\varphi^{n}\right)$ ou plus généralement des suites de la forme $\left(c \varphi^{n}\right)$ avec $c \geq 1$.

(3) Fixons deux entiers positifs $\alpha$ et $\beta$ tels que $\alpha^{2}+\beta$ ne soit pas un carré d'entier et considérons la relation de récurrence $a_{n+2}=2 \alpha a_{n+1}+\beta a_{n}$. Les racines de son polynôme caractéristique sont $\xi=\alpha+\sqrt{\alpha^{2}+\beta}$ et $\eta=\alpha-\sqrt{\alpha^{2}+\beta}$. Par hypothèse, $\xi>1$ est irrationnel et $|\eta|<\xi$. Si $\left(a_{n}\right)_{n \geq 0}$ satisfait cette relation et si

$$
\inf _{n \geq 0} \frac{a_{n}}{\left(\alpha+\sqrt{\alpha^{2}+\beta}\right)^{n}}>0
$$

alors elle satisfait la loi de Benford par rapport à toute base $b \geq 3$. Remarquons qu'il en est de même de la suite

$$
s_{n}=a_{0}+a_{1}+\ldots+a_{n}
$$

par la remarque de la fin du paragraphe 3. Comme il existe $A>0$ et $B \in \mathbb{R}$ tels que $a_{n}=A \xi^{n}+B \eta^{n}$ pour tout $n$, on a

$$
s_{n}=A \frac{\xi^{n+1}-1}{\xi-1}+B \frac{\eta^{n+1}-1}{\eta-1}=\xi^{n}\left(A \frac{\xi-\xi^{-n}}{\xi-1}+B \frac{\eta^{n+1} / \xi^{n}-\xi^{-n}}{\eta-1}\right) \text {. }
$$


Il est facile de vérifier que la suite $\left(s_{n}\right)_{n \geq 0}$ satisfait également la relation de récurrence suivante:

$$
s_{n+3}=(1+2 \alpha) s_{n+2}+(\beta-2 \alpha) s_{n+1}-\beta s_{n} .
$$

(4) Soit enfin une suite $\left(a_{n}\right)_{n \geq 0}$ satisfaisant la relation $a_{n+3}=a_{n+1}+a_{n}$ avec $a_{0}, a_{1}, a_{2} \geq$ 1 arbitraires. Son polynôme caractéristique est $p(x)=x^{3}-x-1$. Une étude succinte de $p(x)$ montre qu'il admet une unique racine réelle $1<\theta<2$, et la formule de Cardan donne:

$$
\theta=\sqrt[3]{\frac{1+\sqrt{\frac{23}{27}}}{2}}+\sqrt[3]{\frac{1-\sqrt{\frac{23}{27}}}{2}} \approx 1.32472 .
$$

Notons $\omega$ et $\bar{\omega} \in \mathbb{C} \backslash \mathbb{R}$ les deux autres racines de $p(x)$. On a

$$
\begin{aligned}
x^{3}-x-1 & =(x-\theta)\left(x^{2}-2 \operatorname{Re}(\omega) x+|\omega|^{2}\right) \\
& =x^{3}-(2 \operatorname{Re}(\omega)+\theta) x^{2}+\left(2 \operatorname{Re}(\omega) \theta+|\omega|^{2}\right) x-|\omega|^{2} \theta,
\end{aligned}
$$

ce qui implique que $|\omega|^{2} \theta=1$, que $2 \operatorname{Re}(\omega)+\theta=0$ et que $2 \operatorname{Re}(\omega) \theta+|\omega|^{2}=-1$. On en déduit que $|\omega|<1$ et que

$$
\omega=-\frac{\theta}{2}+i \sqrt{\frac{4-\theta^{3}}{4 \theta}}=\frac{1}{2}\left(-\theta+i \sqrt{\frac{3-\theta}{\theta}}\right) .
$$

La suite $\left(a_{n}\right)$ étant manifestement non bornée, elle s'écrit $a_{n}=\alpha \theta^{n}+\beta \omega^{n}+\gamma \bar{\omega}^{n}$ pour $\alpha>0$ et $\beta, \gamma \in \mathbb{C}$ convenables. Il reste à vérifier que $\log _{b}(\theta)$ est irrationnel pour tout $b \geq 3$; cela montrera que la suite $\left(a_{n}\right)$ satisfait la loi de Benford par rapport à toute base $b \geq 3$. Or, $x^{3}-x-1$ est le polynôme minimal de $\theta$ : en effet, si ce n'était pas le cas, $\theta$ serait de degré 1 ou 2. Mais cela impliquerait que $x^{3}-x-1$ admettrait au moins une racine rationnelle, ce qui n'est pas le cas.

\section{Référence}

[1] Arnold, V.A.; Avez, A.: Ergodic problems of classical mechanics. W.A. Benjamin, Inc., New York 1968.

[2] Hahn, F.J.: Affine transformations of compact Abelian groups. Amer. J. Math. 85 (1963), 428-446.

[3] Hill, T.: Le premier chiffre significatif fait sa loi. La Recherche 316 (1999), 72-75.

[4] Parry, W.: Topics in ergodic theory. Cambridge Univ. Press, Cambridge 1981.

[5] Weyl, H.: Über die Gleichverteilung von Zahlen mod 1. Math. Ann. 77 (1916), 313-352.

Paul Jolissaint

Institut de Mathématiques

Université de Neuchâtel

Emile-Argand 11

CH-2000 Neuchâtel, Suisse

e-mail: paul.jolissaint@unine.ch 